\begin{tabular}{cc}
\hline & \\
& $\begin{array}{l}\text { International Journal of Case Reports } \\
\text { (ISSN:2572-8776) }\end{array}$
\end{tabular}

\title{
Individualized approach for Coarctation of the Aorta in a Patient with PHACE syndrome and Extensive Hemangiomas
}

\section{Wejdan Ba-Atiyah, Riad Abou Zahr, Amjad Kouatli}

Department of Pediatrics, Pediatric Cardiology section, King Faisal Specialist Hospital and Research Center, Jeddah, Saudi Arabia.

\section{ABSTRACT}

PHACE syndrome is a neurocutaneous disorder with large facial segmental hemangiomas associated with anomalies of the brain, eye and heart.

We report a case of a 3 years old girl who presented at the age of 6 weeks with severe coarctation of the aorta and aortic arch aneurysm. In addition, she had extensive strawberry hemangiomas over the scalp, neck and chest which, after extensive investigation, led to the diagnosis of PHACE syndrome.

After complete evaluation we successfully initiated treatment with propranolol that was pursued without complications over three years with striking success and remarkable regression of her hemangiomas. Meanwhile the coarctation was managed conservatively.

This case highlights that propranolol treatment, while effective against hemangiomas, may contribute to slow the progression of aortic arch aneurysm and delay the need for high risk surgical intervention.

Keywords: PHACE syndrome, Propranolol, Coarctation of the aorta
${ }^{*}$ Correspondence to Author:

Wejdan Ba-Atiyah

Department of Pediatrics, Pediatric Cardiology section, King Faisal Specialist Hospital and Research Center, Jeddah, Saudi Arabia.

How to cite this article:

Wejdan Ba-Atiyah, Riad Abou Zahr, Amjad Kouatli. Individualized approach for Coarctation of the Aorta in a Patient with PHACE syndrome and Extensive Hemangiomas. International Journal of Case Reports, $20204: 128$

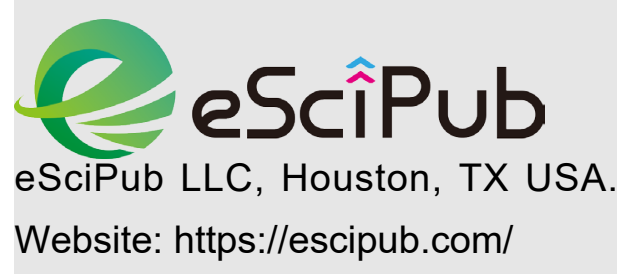




\section{Introduction:}

PHACE syndrome is a neurocutaneous condition, an acronym for posterior fossa malformations, hemangiomas, arterial anomalies, cardiac anomalies, and eye anomalies ${ }^{1}$. Management calls for a multidisciplinary approach including medical therapy for the hemangioma and attention to other involved organs. In patients with PHACE syndrome, $37 \%$ of cases with aortic arch anomalies will need surgical intervention. Nonsurgical cases should be followed with an annual echocardiogram due to the possibility of lesion progression ${ }^{2}$.

\section{Case:}

A 3 years old girl, third child to non-consanguineous parents, was born after an uneventful pregnancy at 38 weeks of gestation via spontaneous vaginal delivery with no postnatal complications. At the age of 14 days, she developed a reddish papule over the scalp which ran an extensive progressive course to involve almost half of the head, neck and chest with progressive swelling of the left side of the neck and she started to develop mild stridor (Figure 1). The family sought medical advice in an outside facility and upon examination a cardiac murmur was noted. Echocardiogram was performed and showed severe coarctation of the aorta. Subsequently, she was referred to our hospital for further management.

On physical examination the patient had no dysmorphic features. She had an audible stridor. There was a massive well defined bright red soft, lobulated, non-translucent, compressible, and partially blanchable swelling over the face, occiput, neck, upper lip, involving both eyelids and occluding the left eye completely. Cardiovascular examination revealed a 4 limb cuff blood pressure of: Right Arm 128/68, Right Leg 73/47, Left Arm 81/52, Left Leg 71/47 with gradient of $50 \mathrm{mmHg}$ between right upper and lower limb. A $2 / 6$ ejection systolic murmur was heard over the right upper sternal border that radiated to the back. Femoral pulses were not palpable. Ophthalmology examination showed no eye involvement. The reminder of her examination was unremarkable.
Clinical findings suggested a differential diagnosis of infantile hemangioma with PHACE syndrome. So further work up was done. Routine hematological investigations were normal. Echocardiogram demonstrated a tortuous aneurysmal transverse aortic arch with a discrete juxtaductal coarctation with mild long segment hypoplasia of the proximal descending aorta with peak gradient of $70 \mathrm{mmHg}$ and moderate left ventricular hypertrophy (Figure 2). Cardiac CT scan revealed a tortuous aneurysmal transverse aortic arch with long segment coarctation at the aortic isthmus. The descending thoracic aorta was unremarkable (Figure 3). CT angiography of the chest demonstrated extensive hemangiomas involving the left side of the neck and extending on the left side of the face into the mediastinum as well as crossing onto the right side and causing tracheal compression (Figure 4). Abdominal ultrasound showed a small mass in the liver suggestive of hemangioma. CT brain revealed evidence of a large mass surrounding the occiput with significant enhancement within the posterior fossa. Eventually, our investigations led to diagnose her with PHACE syndrome based on the presence of an extensive strawberry hemangioma over the scalp, neck and chest with Aortic arch anomaly (Coarctation of the aorta and aneurysm).

The case was discussed in a multidisciplinary meeting that included neurosurgery, vascular surgery, cardiothoracic surgery, pediatric cardiology and neurology services. Due to the extensive hemangiomas that were compressing her airway, she was deemed high risk for intubation, general anesthesia, and coarctation repair. So the decision was made after reviewing the available literatures to manage conservatively and start propranolol. Oral propranolol $1 \mathrm{mg} / \mathrm{kg} / \mathrm{day}$ in 4 divided doses was started and gradually titrated up to $2 \mathrm{mg} / \mathrm{kg} /$ day under close observation in the hospital over 2 weeks after which the patient was discharged home in stable condition. During outpatient visits, we were doing annual follow up with echo and cardiac MRI and there 
was a dramatic gradual regression in the hemangiomas and without progression of the aortic coarctation (Figure 5). No propranolol side effects were reported. At 3 years of age, her 4 limb cuff blood pressure was: Right Arm 118 / 90, Right Leg 105 / 69, Left Leg 95 / 61 , Left Arm 101 /
77. Cardiac MRI evaluation at the time demonstrated tortuous stable size aneurysmal aortic arch with moderate long segment coarctation of aorta (Figure 6). Echocardiogram documented a peak gradient of $45-50 \mathrm{mmHg}$ and mild LVH.
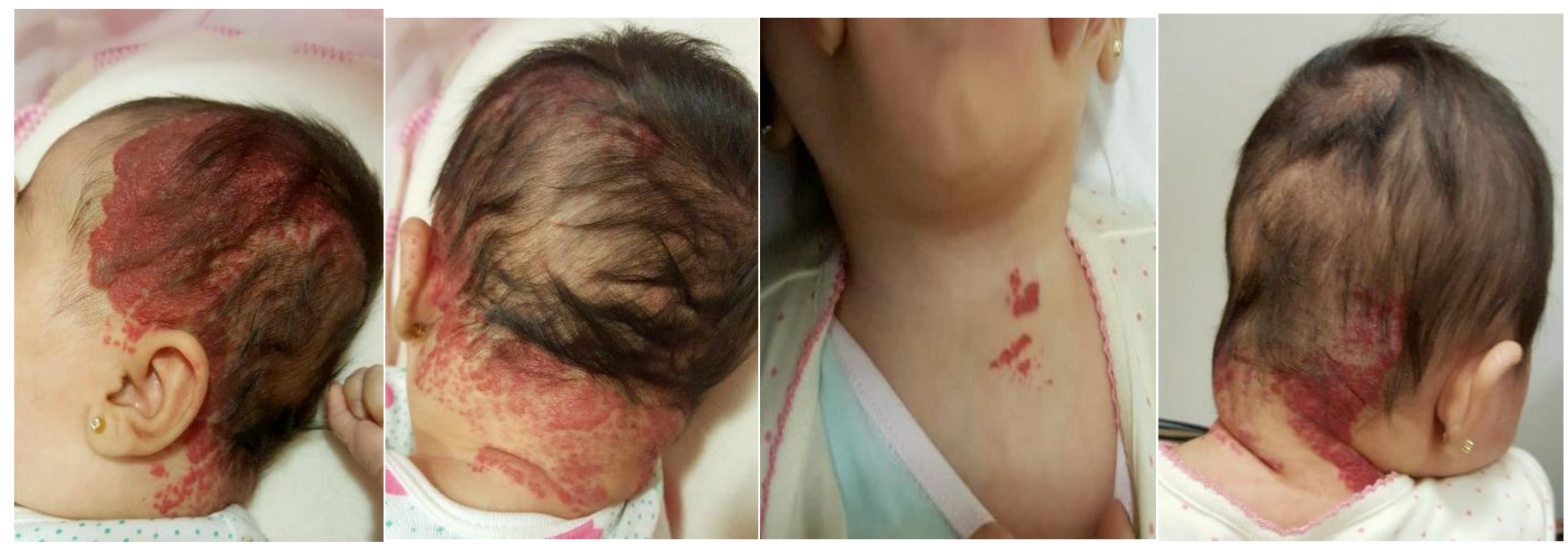

Figure 1: Pictures demonstrate the extensive hemangioma of the head and neck before treatment.

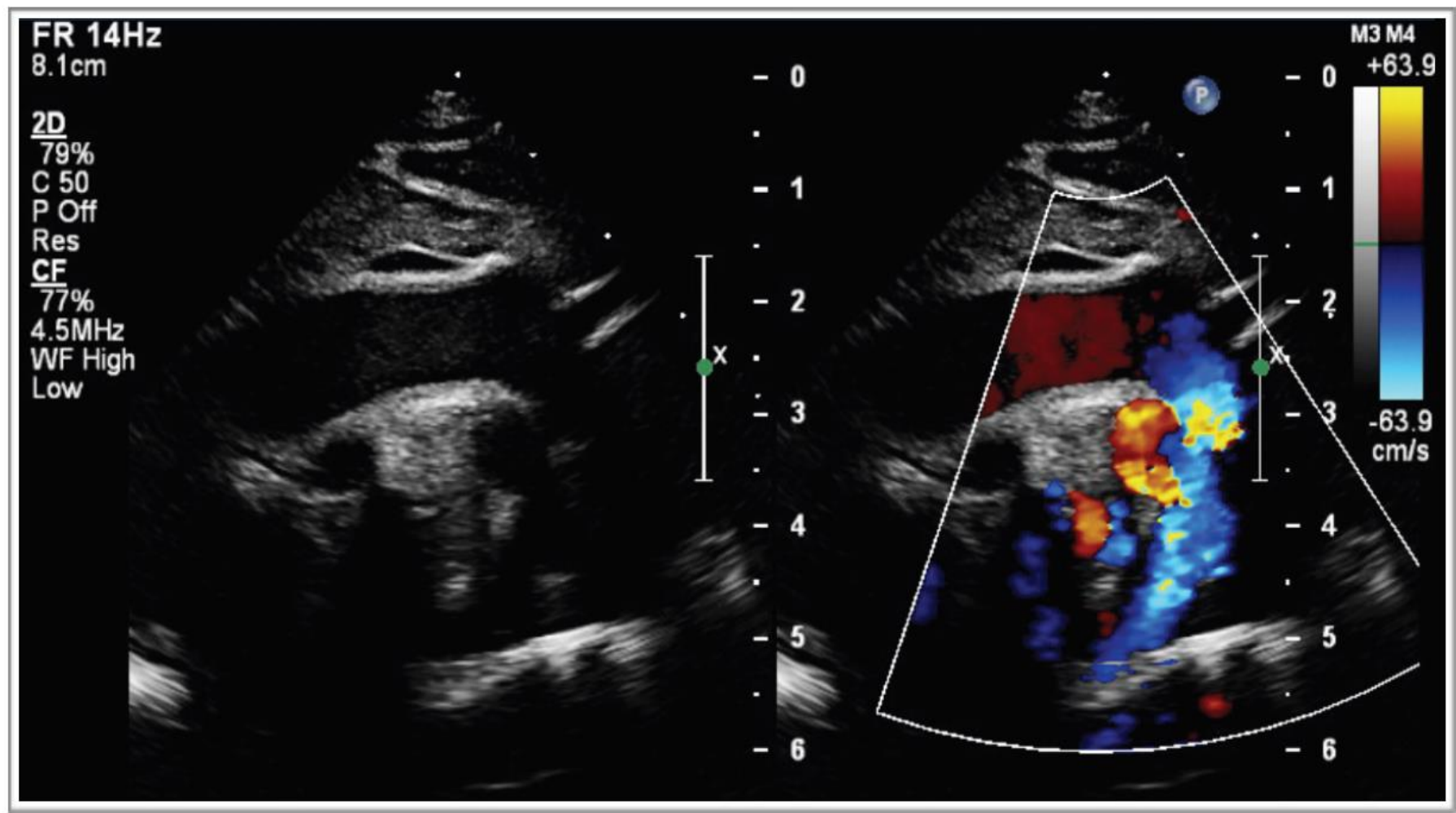

Figure 2 : Suprasternal view in 2D echocardiogram demonstrate Discrete Juxutaductal coarctation with mild long segment hypoplasia of proximal descending aorta with a round shadow just left to coarctation segment with blood swirling in it . 


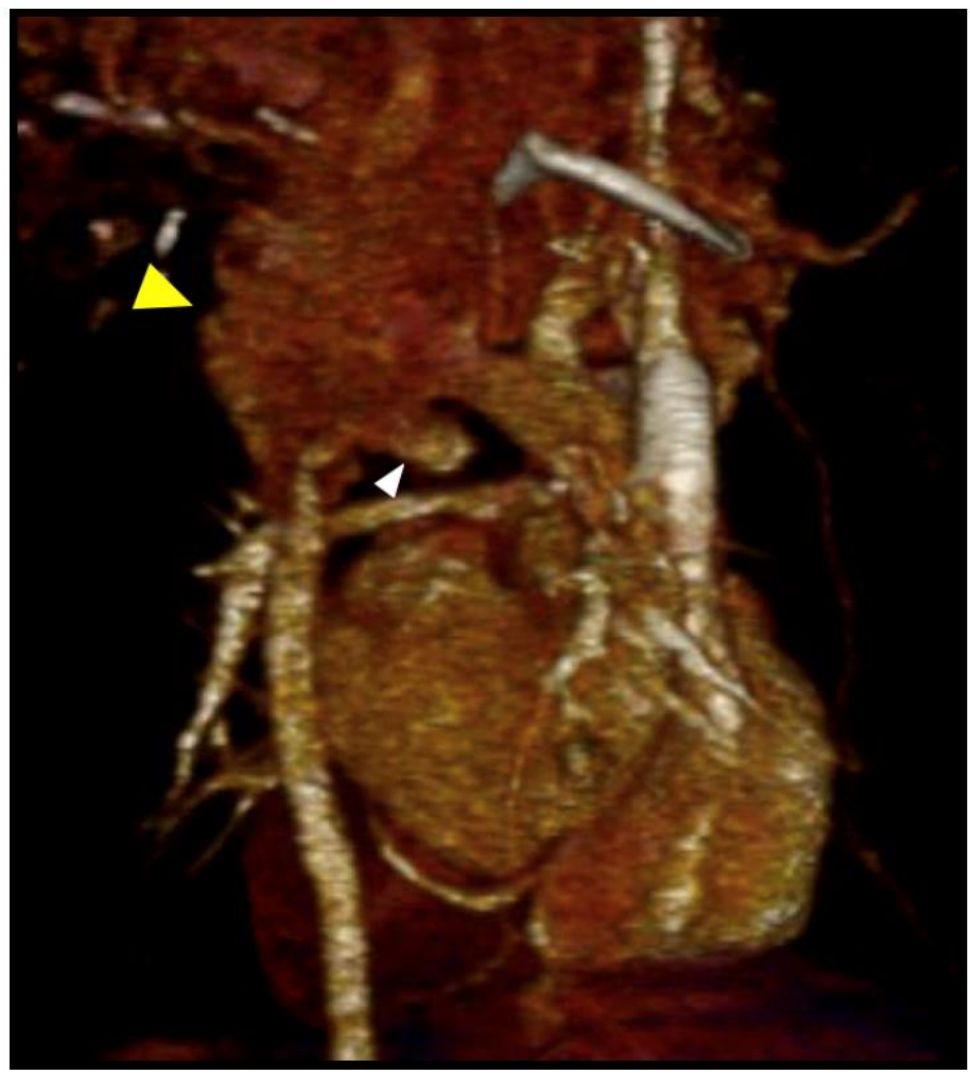

Figure 3: 3D volume rendering from CT angiography in a sagittal oblique plane demonstrates tortuous aneurysmal transverse arch (white arrow) with coarctation. Note contrast uptake by extensive hemangioma in the neck and mediastinum region (yellow arrow) obscuring part of the transverse arch.

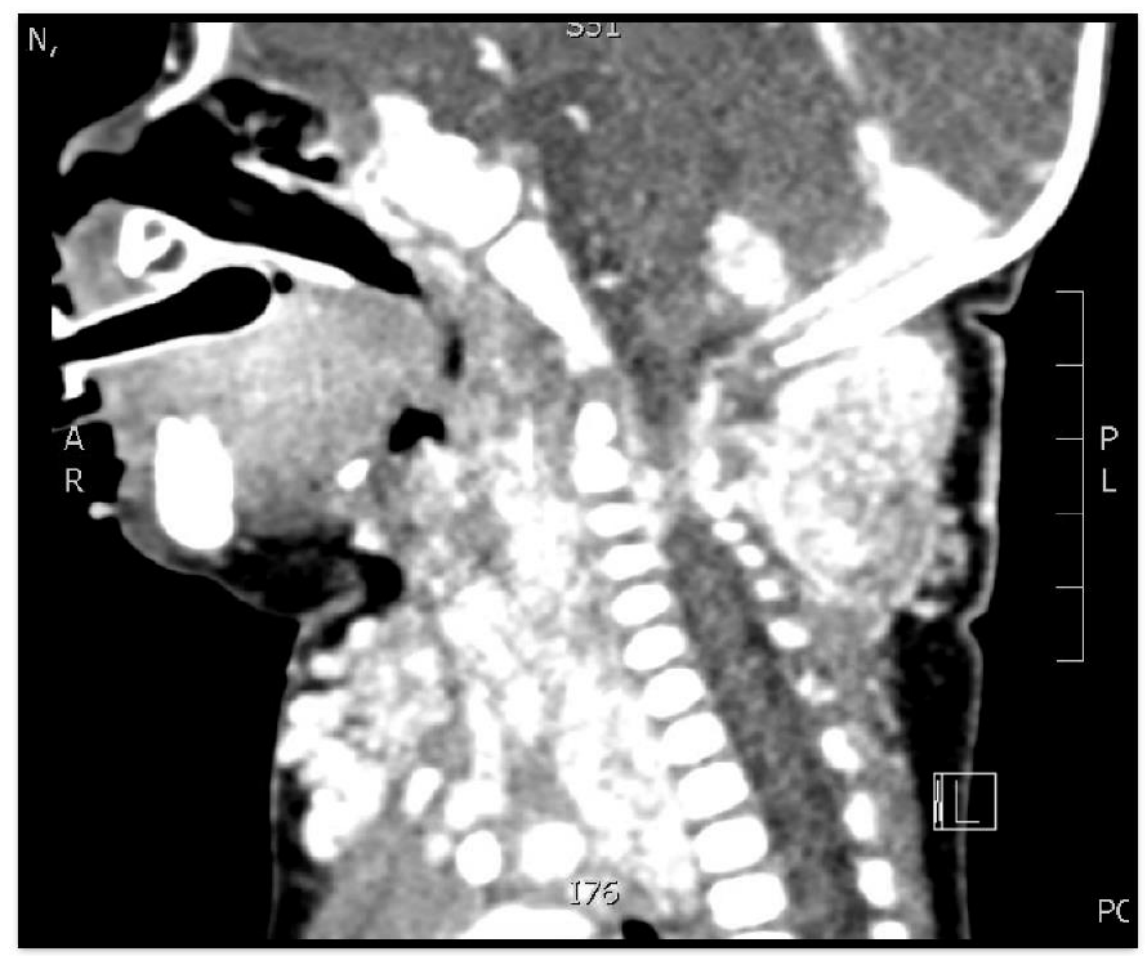

Figure 4 : CT angiography of the chest demonstrated extensive hemangiomas involving extending to the mediastinum causing tracheal compression . 


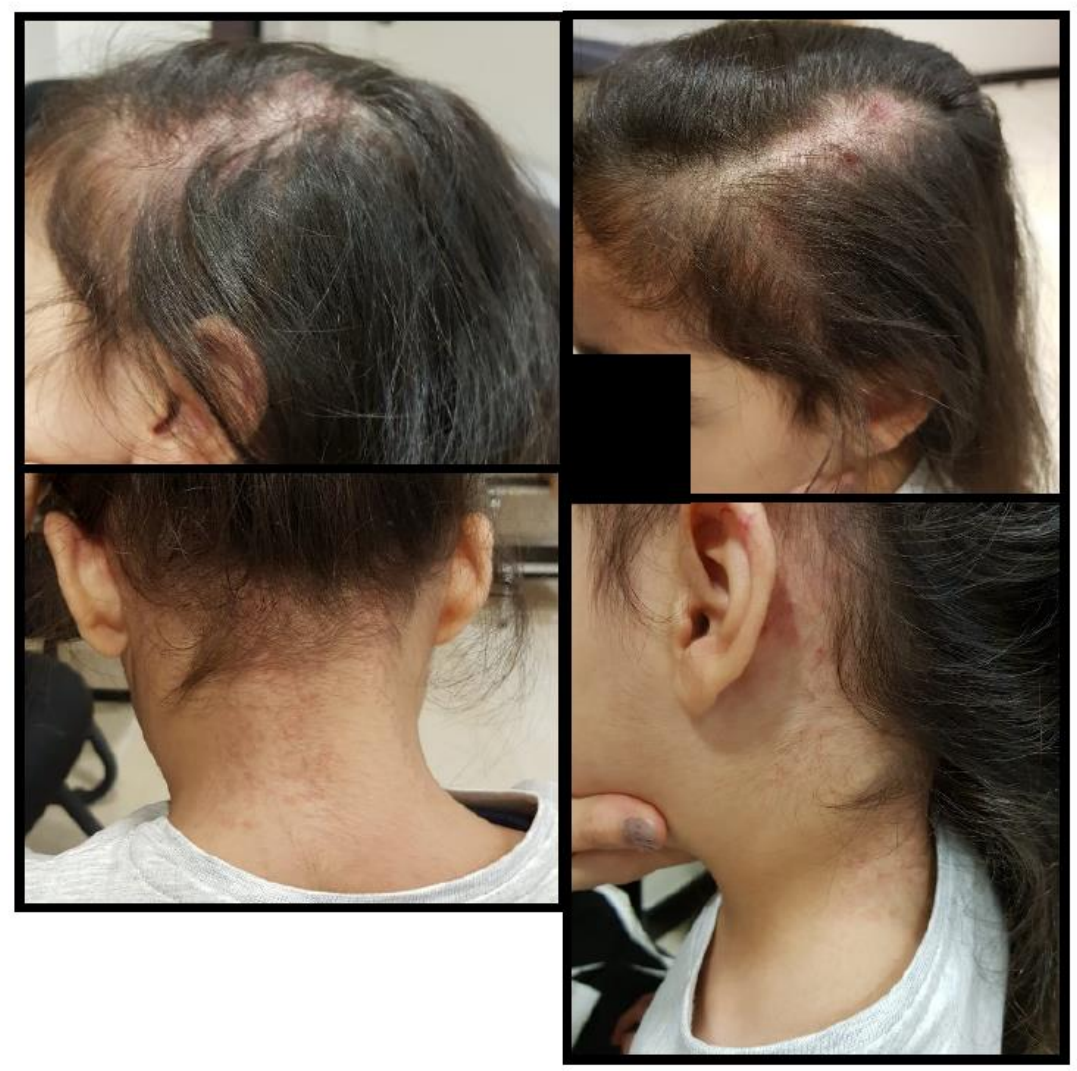

Figure 5 : Pictures demonstrate small scattered residual hemangioma after treatment with propranolol

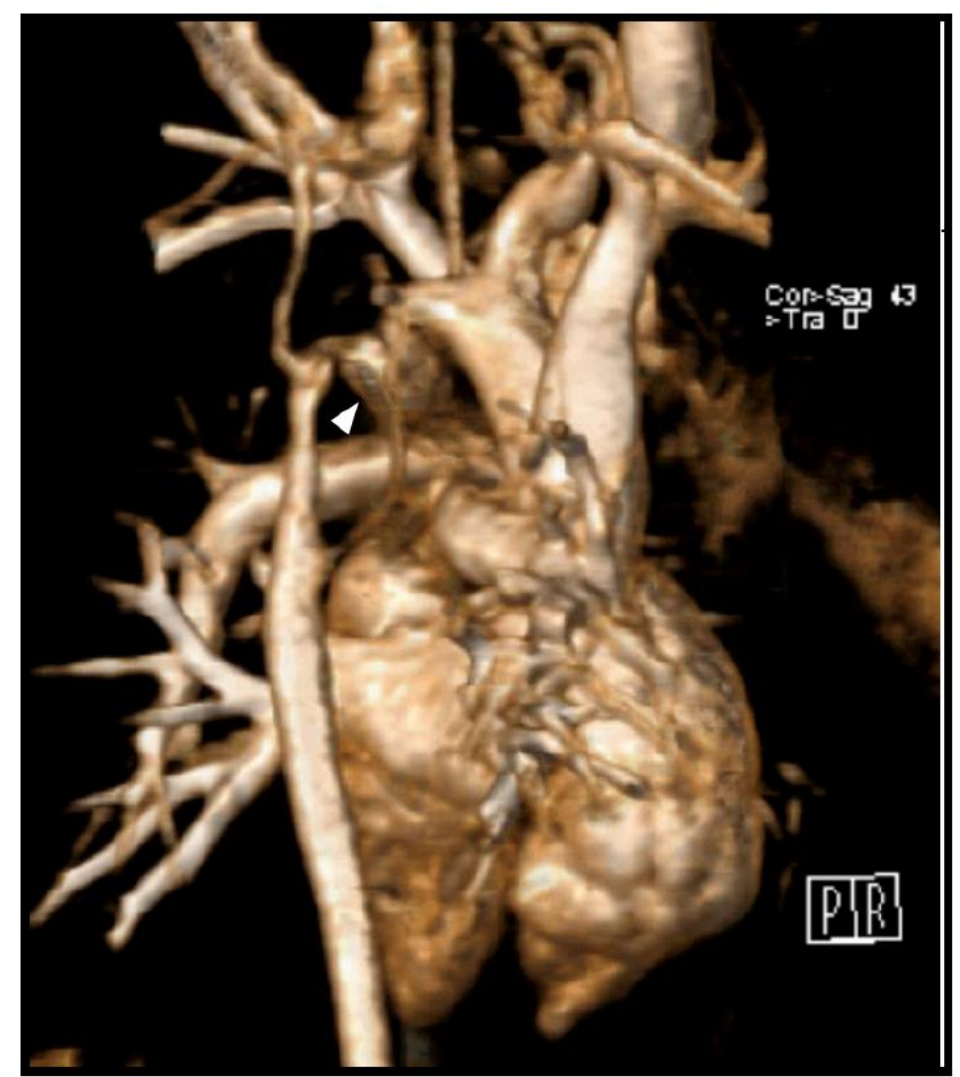

Figure 6 : 3D volume rendering from magnetic resonance angiography (MRA) in a sagittal oblique plane demonstrates tortuous aneurysmal transverse arch (white arrow ) with long segment coarctation of the aorta . Note resolution of the previously described hemangioma. 


\section{Discussion:}

PHACE syndrome is a neurocutaneous condition that involves posterior fossa malformations, hemangiomas, arterial anomalies, cardiac anomalies and eye anomalies ${ }^{1}$. The pathogenesis of PHACE syndrome is unknown. There is no evidence of genetic abnormalities that contribute to the development of PHACE syndrome ${ }^{3}$. It has been postulated to result from defective embryogenesis between weeks 3 and 12 of gestation, before or during vasculogenesis, because certain malformations and $\mathrm{IH}$ can affect the same side of the body ${ }^{4}$. The first description of the association of PHACE syndrome with brain abnormalities was reported in 1978 by PascualCastro- viejo ${ }^{5}$. Over 300 cases of PHACE syndrome have been reported, and it is considered one of the most common neurocutaneous vascular disorders in childhood ${ }^{6}$. The prevalence of congenital cardiac diseases in patients with PHACE syndrome is $41 \%$ to $67 \%$, with coarctation of the aorta comprising $19-30 \%$ of these cases $^{7}$. In 2009, a diagnostic criteria for PHACE syndrome was established ${ }^{8}$ and subsequently revised in 2016 by Garzon et al ${ }^{2}$. The criteria for the diagnosis of PHACE syndrome, stratifying it into 2 main categories:

1. PHACE syndrome, defined by the presence of segmental infantile hemangioma larger than $5 \mathrm{~cm}$ on the face, scalp, or cervical region, associated with 1 major criterion or 2 minor criteria.

2. Possible PHACE syndrome, defined by the presence of infantile hemangioma and $1 \mathrm{mi}-$ nor criterion.

Major and minor criteria have been determined, based on the vascular and structural involvement of the brain, cardiovascular system, eye, and midline ${ }^{8}$.

Patients with PHACE syndrome tend to have long segment of aortic stenosis in the mid-transverse arch, arch aneurysms, aberrant origin or stenosis of head and neck vessels, and increased tortuosity. All of these features make surgical planning and intervention challenging ${ }^{9}$.
The management depends on the affected organ systems and requires different specialties including dermatology, ophthalmology, pediatrics, cardiology and neurology ${ }^{8}$. Propranolol is the preferred medication for infantile hemangioma when systemic treatment is indicated ${ }^{10}$. The proposed mechanism of action of propranolol on hemangiomas include the control of hypoxic stress with upregulated hypoxia inducible factor 1 (HIF-1), apoptosis induction, and decreased production of vascular endothelial growth factors (VEGF) and fibroblastic endothelial growth factors (FGF- $\beta$ ) ${ }^{11}$. The safe use of propranolol in patients with PHACE syndrome is based on examination of extracutaneous anomalies and recommendations of a multidisciplinary team.

In patients with PHACE syndrome with aortic arch anomalies, $37 \%$ of cases will need surgical intervention. Nonsurgical cases should be followed with echocardiography due to the possibility of lesion progression ${ }^{2}$. Extensive aortic arch reconstruction is commonly required in children with PHACE syndrome and coarctation due to the bizarre nature of the obstruction. A retrospective, observational review was carried out at Children's Hospital of Wisconsin in Milwaukee, United States where seven patients with PHACE required aortic arch reconstruction from 1996 to 2015 12. All needed complex surgical approaches (4 conduit grafts, 2 patch aortoplasties, 1 subclavian flap) to relieve the obstruction because of long-segment transverse and proximal descending aortic arch dysplasia with areas of stricture and aneurysmal dilation. 3 children, who had surgery after age 1 , showed significant progression of the arch obstruction and/or adjacent aneurysmal segment dilation over time. No deaths or perioperative complications were reported. 5 patients had cerebrovascular arterial dysplasia. Recurrent arch obstruction developed in 3 patients at an intermediate follow-up interval of 6.2 years ${ }^{12}$. The higher incidence of recurrent aortic arch obstruction in PHACE syndrome is likely related to abnormalities within the aortic wall $9,12,13$. 


\section{Conclusion:}

We conclude that this case report highlights that propranolol treatment in patient with PHACES syndrome can help in improvement of extensive large hemangioma and at the same time may help in stabilizing the aortic aneurysm and eventually delaying the need for high risk surgical intervention with promising results.

\section{References}

1. Frieden IJ, Reese V, Cohen D. PHACE syndrome. The association of posterior fossa brain malformations, hemangiomas, arterial anomalies, coarctation of the aorta and cardiac defects, and eye abnormalities. Arch Dermatol. 1996;132(3):30711.

2. Garzon MC, Epstein LG, Heyer GL, Frommelt PC, Orbach DB, Baylis AL, et al. PHACE Syndrome: Consensus-Derived Diagnosis and Care Recommendations. The Journal of pediatrics. 2016;178:24-33 e2.

3. Raas-Rothschild A, Nir A, Gillis R, Rein AJ. Giant congenital aortic aneurysm with cleft sternum, supraumbilical raphe, and hemangiomatosis: report and review. Am J Med Genet. 2000;90(3):243-5.

4. Haggstrom AN, Lammer EJ, Schneider RA, Marcucio R, Frieden IJ. Patterns of infantile hemangiomas: new clues to hemangioma pathogenesis and embryonic facial development. Pediatrics. 2006;117(3):698-703.

5. Pascual-Castroviejo I. Vascular and nonvascular intracranial malformation associated with external capillary hemangiomas. Neuroradiology. 1978;16:82-4.

6. Rotter A, Samorano LP, Rivitti-Machado MC, Oliveira ZNP, Gontijo B. PHACE syndrome: clinical manifestations, diagnostic criteria, and management. An Bras Dermatol. 2018;93(3):40511.

7. Haggstrom AN, Garzon MC, Baselga E, Chamlin SL, Frieden IJ, Holland K, et al. Risk for PHACE syndrome in infants with large facial hemangiomas. Pediatrics. 2010;126(2):e418-26.

8. Metry D, Heyer G, Hess C, Garzon M, Haggstrom A, Frommelt $P$, et al. Consensus Statement on Diagnostic Criteria for PHACE Syndrome. Pediatrics. 2009;124(5):1447-56.

9. Castellano-Martinez $A$, Rodriguez-Gonzalez $M$, Benavente-Fernandez I, Zuazo-Ojeda A. Hemangioma, aortic coarctation and intracranial dolichoectasia: PHACE syndrome. Pediatr Int. 2017;59(2):230-3.
10. Drolet BA, Frommelt PC, Chamlin SL, Haggstrom A, Bauman NM, Chiu YE, et al. Initiation and use of propranolol for infantile hemangioma: report of a consensus conference. Pediatrics. 2013;131(1):128-40.

11. Leaute-Labreze C, Dumas de la Roque E, Hubiche T, Boralevi F, Thambo JB, Taieb A. Propranolol for severe hemangiomas of infancy. N Engl J Med. 2008;358(24):2649-51.

12. Caragher SP, Scott JP, Siegel DH, Mitchell ME, Frommelt PC, Drolet BA. Aortic arch repair in children with PHACE syndrome. J Thorac Cardiovasc Surg. 2016;152(3):709-17.

13. Prada F, Mortera C, Bartrons J, Rissech M, Jimenez L, Carretero J, et al. Complex aortic coarctation and PHACE syndrome. Rev Esp Cardiol. 2010;63(11):1367-70.

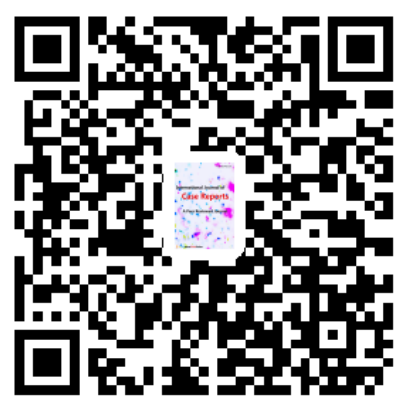

